\title{
$1 \quad$ Optimal Operation of Stationary and Mobile Batteries in Distribution Grids
}

\author{
Yubo Wang ${ }^{a^{*}}$, Wenbo Shi ${ }^{\mathrm{b}}$, Bin Wang ${ }^{\mathrm{a}}$, Chi-Cheng Chu ${ }^{\mathrm{a}}$ and Rajit Gadh ${ }^{\mathrm{a}}$
}

$3 \quad{ }^{a}$ Department of Mechanical Engineering, UCLA, Los Angeles, United States

$4 \quad{ }^{b}$ Center for Green Buildings and Cities, Harvard University, Cambridge, United States

5 * Corresponding author. Address: 44-120 Engr. IV, 420 Westwood Plaza, Los Angeles, CA 90095, USA. Email:

6 ybwang@ucla.edu

\section{Abstract}

8 The trending integrations of Battery Energy Storage System (BESS, stationary battery) and Electric

9 Vehicles (EV, mobile battery) to distribution grids call for advanced Demand Side Management (DSM)

10 technique that addresses the scalability concerns of the system and stochastic availabilities of EVs.

11 Towards this goal, a stochastic DSM is proposed to capture the uncertainties in EVs. Numerical

12 approximation is then used to make the problem tractable. To accelerate the computational speed, the

13 proposed DSM is tightly relaxed to a convex form using second-order cone programming. Furthermore,

14 in light of the continuous increasing problem size, a distributed method with a guaranteed convergence

15 is applied to shift the centralized computational burden to distributed controllers. To verify the

16 proposed DSM, real-life EV data collected on UCLA campus is used to test the proposed DSM in an IEEE

17 benchmark test system. Numerical results demonstrate the correctness and merits of the proposed 18 approach.

\section{Keywords}

20 BESS; DSM; distributed optimization; EV; stochastic optimization

\section{1. Introduction}

22 Coupled with the need for better demand-supply balance, future smart grid represents a vision to 23 incorporate an increasing number of storage devices in distribution grids [1]. Facilitated by recent 24 advances in Demand Side Management (DSM), chemical [3], thermal [4] and mechanical [5] storage 25 systems are optimally managed in response to electricity price incentives. Combined the with the target 26 of cutting down the overall operation cost, DSM collaboratively addresses peak shaving and power 27 quality improvements with cyber physical constraints [5]-[7]. 
The increasing number of Electric Vehicles (EVs) integrated to distribution grids introduces new challenges as well as opportunities [8]. On one hand, unregulated charging creates sharp peaks in addition to the original load profile [9]-[10]. On the other hand, with the introduction of Vehicle-to-Grid (V2G), EV switches its role from heavy load to distributed virtual generator [11]-[12]. Therefore, DSM in distribution grids that jointly considers the potentials of both traditional stationary storage and EVs has been a subject of significant ongoing research.

Early works have primarily assessed managing the EVs as stationary storages. Extensive studies have been performed assuming the perfect knowledge of EVs' availabilities [13]-[15]. However, unlike stationary storages, one of the key features of EVs relies on the uncertainty in their mobility: the EV owners are not committed to pre-defined schedules. The randomness in EV arrival time, leave time and energy demands makes the deterministic DSM less capable of managing stochastically behaved EVs. Though a conceptual incentive program has been designed in [16] for locking down the uncertainty, the urgent need for addressing the uncertainty under established tariff model still widely exists. As an alternative, stochastic behaviors of EVs have been studied using robust optimization [17] and two-stage stochastic programming in [18]. See also [19] for event-based V2G scheduling formulation. These works focused on storage management using a lumped model: the power balance was maintained in one single node, assuming the node to be an isolated from the other nodes on the power system.

Turning attention to the distribution grids, buses are connected by power line and interact with each other under power flow constraints. The isolated lumped model may turn out to either violate the bus voltage limits or result in excessive power losses in the power lines. Consequently, Voltage Regulation (VR) and Optimal Power Flow (OPF) are the two important topics capturing researchers' interests in distribution grids. A number of centralized methods have been proposed including particle swarm optimization [20], genetic algorithm [21], mixed integer non-linear programming [22], neural networks [23] and fuzzy logic [24]. These methods require expensive computational power especially when the system grows large. Furthermore, when taking EV management into account, the DSM needs to know the availabilities of EVs in order to achieve the optimal performance of the system, raising privacy concerns from the EV owners [25].

When dealing with fast growing controllable distributed generations and storages in distribution grids, it 56 is natural to apply distributed algorithms. A number of recent studies have explored the applications of distributed algorithms in DSM. [26], [27] are formulated based on dual decomposition of a convex 
convex OPF problem into DC power flow model. In order to guarantee the dual decomposition converges to its original optimum, the formulation of problem has to be convex [28]. Researchers in [29]-[31] have taken into account the OPF problem in a distributed way, however, did not capture the uncertainties lied in the system.

In this context, this paper presents the DSM in distribution grids under both deterministically and stochastically available resources. Battery Energy Management System (BESS) is chosen as the representative of the existing controllable load/resource that has full availability throughout the entire day. On the other hand, EVs as mobile storage devices, represent the widely existing power system uncertainties. The objective is to minimize the nodal operational cost and entire power losses in distribution grids while satisfying bus voltage regulations and power flow constraints. Specifically, we formulate the OPF problem by convex relaxation for radial distribution grids. See [32], [33] for secondorder cone programing based relaxation and [34] for semidefinite programing based relaxation. Sufficient condition for the tightness of the convex relaxation holds under three restrictions: the network has to be radial; the power injection to each bus cannot be too large; and bus voltages are kept around nominal values. These restrictions hold for most of the real-life distribution grids. On the other hand, stochastic optimization is applied to model the uncertainties in EVs, which exists in arrival time, departure time and energy demands. A model-free Sample Average Approximation (SAA) method [35] is employed to make the stochastic modeling tractable. Furthermore, to manage the distribution grids in distributed fashion, Alternating Direction Method of Multipliers (ADMM) [28] is used to solve the problem. As the ADMM based DSM does not need to have the knowledge of end-user, their privacies are also preserved.

The technical contribution of this paper is three-fold: 1 ) the proposed DSM accounts for both the nodal operational cost and OPF over the distribution network. Through tight relaxation, the original problem is further formulated into convex optimization, which can be effectively solved with solvers for guaranteed convergence.2) The uncertainties rely in EV arrival time, departure time and energy demands are modeled with stochastic programming and model-free approximation, which are later verified by reallife data collected from 19 EV users on UCLA campus over one year period. 3) An ADMM based distributed method is applied to solve for the proposed DSM. It makes the DSM scalable and end-user privacy-preserving, taking one step closer to practical industrial application. The distributed solver is verified numerically on an IEEE 13-bus benchmark test system, followed by comprehensive discussions on the observations. 
The remaining of this paper is organized as follows: Section 2 introduces the formulation of the DSM

91 problem in distribution grids. Section 3 develops the stochastic modeling of EVs. Subsequently, Section 4 explains the convex relaxation of the original problem and distributed algorithm. Numerical verifications are performed in Section 5 and conclusions are drawn in Section 6.

\section{System Modeling}

95 In this section, we describe the overall system architecture, and the modeling of the deterministic elements in the system, bearing the uncertainty elements in mind (in Section 3). The deterministic elements include DSM model, BESS model, and distribution grids model.

\subsection{System Architecture}

As shown in Fig.1, the studied system is in distribution grids connected to a substation at the root node. Without loss of generality, under each bus, it connects a set of BESSs, a number of EVs and uncontrollable loads. There are two major players in the distribution grids, namely Distribution System Operator (DSO) and Sub-Distribution System Operator (SDSO). The DSO is in charge of managing the whole distribution grids and the SDSO is managing the BESSs, EVs and loads under each bus. There exists two-way communication links between DSO and SDSO. In this paper, we consider a slotted model and slice the timeframe of a day into $H$ equal time slots. The time is represent as $t$, and $t \in\{1,2, \ldots, H\}$. The set of $\{t \mid t \in\{1,2, \ldots, H\}\}$ is denoted as $\boldsymbol{T}$. As the target of this paper is to manage the distribution grids under steady-state, we assume the power flow stays the same between each time slot.

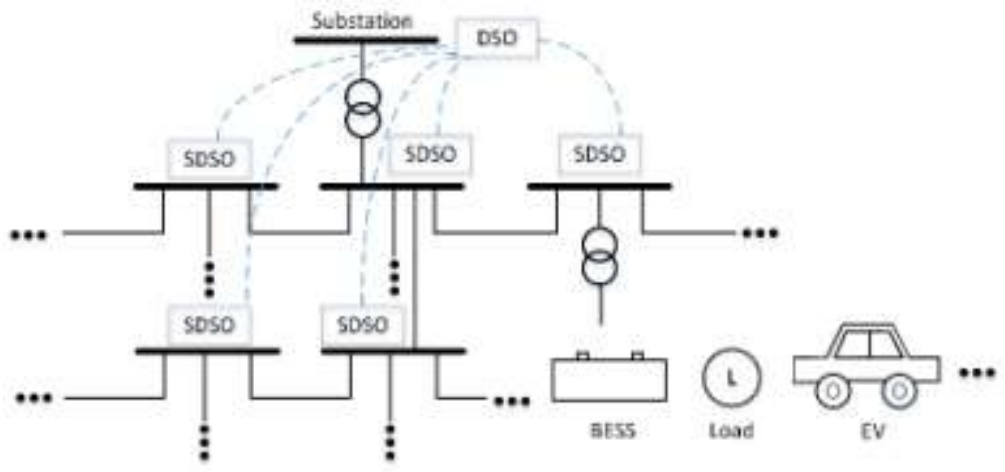

Fig.1. Studied distribution grids architecture

2.2. DSM Model

111 For notation: $\overline{(\cdot)}$ represents the upper limit of a scaler and similarly $(\cdot)$ represents the lower limit. $(\cdot)^{*}$ 112 stands for the conjugate of a scaler. For the same variable, capital letter is used to denote real variables, 
small letter is used to denote complex variables. The imaginary unit is denoted as $\boldsymbol{j}$. Other bold symbols represent sets. Finally, the expectation is denoted by $\mathbb{E}[\cdot]$.

The objective of the DSM proposed in this paper is to minimize the overall nodal operation cost and power losses in distribution line while keeping the bus voltages regulated in the acceptable range. The distribution grids are represented as a connected graph $G=(\boldsymbol{N}, \xi)$. Let $i \in N=\{1,2, \ldots, N\}$ denote the node (bus) of the distribution grids, and $\xi$ denote the lines. We use bus 1 to represent the root node shown in Fig.1. If there is a line $(i, j) \in \xi$ connecting bus $i$ and bus $j$, let $z_{i j}=R_{i j}+j X_{i j}$ be the line impedance, and $i_{i j}(t)$ be the line current from $i$ to $j$ at time $t, P^{i}(t)$ and $Q^{i}(t)$ are the active and reactive power drawn at bus $i$ at time t, and the apparent power drawn at bus $i \in \boldsymbol{N}$ is represented as $s^{i}(t)=P^{i}(t)+j Q^{i}(t)$. The positive sign for $s^{i}(t)$ represents power drawn from bus $i$. For each bus $i$, there exists a set of BESSs, denoted $\boldsymbol{M}_{b}^{i}$, and a set of EVs, denoted $\boldsymbol{M}_{e}^{i}$. Let $\Pi(t) \in \mathfrak{R}^{1+}$ be the energy price for a studied day, the DSM objective function is then presented as follows:

$$
\min \sum_{t} \Pi(\mathrm{t}) \sum_{i \in N \backslash\{1\}} P^{i}(t)+\gamma \sum_{t} \sum_{(i, j) \in \xi} R_{i j}\left|i_{i j}(t)\right|^{2}
$$

The DSM objective is the summation of two parts: the first part minimizes the nodal operational cost, and the second part minimizes the power losses over distribution line. The two parts are connected by a weighting factor $\gamma$. For simplicity, the time constant for each time slot is neglected, which does not affect the optimum of the system.

We present the overall architecture of the proposed DSM in Fig.2. We first model the BESS and power propagation in Section 2.3 and 2.4. EVs are modeled taking into consideration of their stochastic nature. The development of EV modeling, together with PDF estimation and numerical approximation is detailed in Section 3, known as stochastic optimization. The combination of BESS, network and EV model becomes the centralized DSM. Its distributed counterpart is developed in Section 4. To ensure the convergence of distributed DSM, a tight convex relaxation is made in Section 4.1. Then, a distributed method is developed in Section 4.2 to dispatch the centralized computational burden to distributed computational nodes. The convergence and method to speed up the convergence is discussed in Section 4.3. 


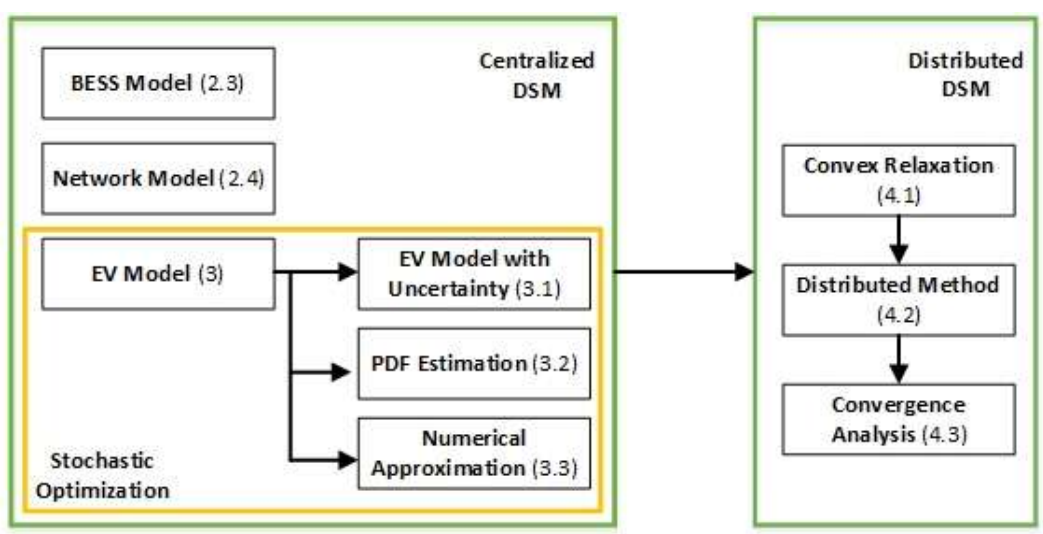

Fig.2. Overall architecture of the proposed DSM

141

\subsection{BESS Model}

142 Let $P_{b}^{i, m}(t)$ and $Q_{b}^{i, m}(t)$ denote the active power and the reactive power output of the BESS $m$ under 143 bus $i$ in time slot $t$. And $S_{b}^{i, m}(t)$ denote the apparent power limit of a physical inverter interfaced with 144 the BESS. The power constraint of the BESS for $\forall i \in N \backslash\{1\}, \forall m \in \boldsymbol{M}_{b}^{i}$ can be represented as follows:

$$
P_{b}^{i, m^{2}}(t)+Q_{b}^{i, m^{2}}(t) \leq S_{b}^{i, m^{2}}(t)
$$

146 This constraint models the maximum power rating of an inverter. The energy constraint of the BESS for $147 \forall i \in \boldsymbol{N} \backslash\{1\}, \forall m \in \boldsymbol{M}_{b}^{i}$ is modeled as:

$$
\underline{E_{b}^{i, m}} \leq E S_{b}^{i, m}+\sum_{t \in \boldsymbol{T}} P_{b}^{i, m}(t) \leq \overline{E_{b}^{i, m}}
$$

149 where $E S_{b}^{i, m}$ represents the initial State-of-Energy (SoE) of the BESS $m$ under bus $i$ at time $t=0 . \overline{E_{b}^{i, m}}$ 150 and $\underline{E_{b}^{i, m}}$ represent the upper and lower limit of the BESS, respectively. The lower bound for Eqn.(2.b) is 151 a positive real scaler to prevent the BESS from deep discharge, which is known to exponentially decrease 152 the lifespan of a battery [37]. After a full day's operation, we would like the SoE of BESS for $\forall i \in$ $153 \boldsymbol{N} \backslash\{1\}, \forall m \in \boldsymbol{M}_{b}^{i}$ to be some pre-defined final value:

$$
E S_{b}^{i, m}+\sum_{t} P_{b}^{i, m}(t)=E F_{b}^{i, m}
$$


155 where $E F_{b}^{i, m}$ is the final SoE of the BESS after a full day's operation.

156

157

158

159

$$
s_{i j}(t)=v_{i}(t) i_{i j}^{*}(t)
$$

160

$$
s_{i j}(t)-z_{i j}\left|i_{i j}(t)\right|^{2}-\sum_{(j, k) \in \xi} s_{j k}(t)=s_{j}(t)
$$

where the bus $i$ voltage is denoted as $v_{i}$, and $s_{i j}(t)=P_{i j}(t)+j Q_{i j}(t)$ denote the apparent power flowing from bus $i$ to bus $j$. The positive sign for $s_{i j}$ is defined as power flowing from $i$ to $j$. Eqn.(3.a) is the Ohm's law, Eqn.(3.b) and Eqn.(3.c) are the definitions for apparent power and power flow, respectively. Substituting Eqn.(3.a) and Eqn.(3.b) into Eqn.(3.c) we will have for $\forall(i, j) \in \xi, j \neq 1$ :

$$
P^{j}(t)=P_{i j}(t)-R_{i j}\left|i_{i j}(t)\right|^{2}-\sum_{(j, k) \in \xi} P_{j k}(t)
$$

166

$$
Q^{j}(t)=Q_{i j}(t)-X_{i j}\left|i_{i j}(t)\right|^{2}-\sum_{(j, k) \in \xi} Q_{j k}(t)
$$

167

$$
\left|v_{i}(t)\right|^{2}-\left|v_{j}(t)\right|^{2}=2\left(R_{i j} P_{i j}(t)+X_{i j} Q_{i j}(t)\right)-\left(R_{i j}^{2}+X_{i j}^{2}\right)\left|i_{i j}(t)\right|^{2}
$$

168

$$
\left|v_{i}(t)\right|^{2}\left|i_{i j}(t)\right|^{2}=P_{i j}^{2}(t)+Q_{i j}^{2}(t)
$$

170 Eqn.(4.a)-(4.d) define the power flow of the distribution grids. In an attempt to model VR, we put the 171 following constraints at each buses: 


$$
\underline{V} \leq\left|v_{i}\right| \leq \bar{V}, \forall i \in N \backslash\{1\} ;\left|v_{1}\right|=V_{c}
$$

$173 \underline{V}$ and $\bar{V}$ are the lower and upper voltage magnitude limits. The bus voltage at root node 1 is assumed to 174 be constant $V_{c}$, which results from the fact that it is connected to the transmission system and be modeled as an infinite bus. The active and reactive power drawn the root node is defined as:

$$
P^{1}(t)=\sum_{j:(1, j) \in \xi} P_{1 j}(t)
$$

176

$$
Q^{1}(t)=\sum_{j:(1, j) \in \xi} Q_{1 j}(t)
$$

178 Putting additional physical capacity constraints on the rating of the substation we have:

$$
\left|P^{1}(t)+j Q^{1}(t)\right| \leq \overline{S^{1}}
$$

The power under each bus for $\forall i \in N \backslash\{1\}$ is defined as follows:

$$
P^{i}(t)=P_{l}^{i}(t)+\sum_{m \in M_{e}^{i}} P_{b}^{i, m}(t)+\mathbb{E}\left[\sum_{m \in M_{e}^{i}} P_{e}^{i, m}\left(\delta^{i, m}, v^{i, m}, \chi^{i, m}, t\right)\right]
$$

$$
Q^{i}(t)=Q_{l}^{i}(t)+\sum_{m \in M_{e}^{i}} Q_{b}^{i, m}(t)+\mathbb{E}\left[\sum_{m \in M_{e}^{i}} Q_{e}^{i, m}\left(\delta^{i, m}, v^{i, m}, \chi^{i, m}, t\right)\right]
$$

183 where $P_{l}^{i}(t)$ and $Q_{l}^{i}(t)$ are the active and reactive power uncontrollable load at time $t$. $P_{e}^{i, m}(t)$ and $Q_{e}^{i, m}(t)$ are the active and reactive power EV $m$ under bus $i$ absorbs. $\delta^{i, m}, v^{i, m}, \chi^{i, m}$ denote the 185 uncertainties in the EV arrival time, departure time and energy demand, respectively. $\mathbb{E}[\cdot]$ denotes the 186 expectation. The value of $P_{e}^{i, m}$ and $Q_{e}^{i, m}$ are subject to the uncertainty in EV. We will develop the EV 187 model in the following section, showing how the uncertainties are modeled in EVs. 


\section{Stochastic EV Modeling}

189

$$
A_{e}^{i, m}\left(\delta^{i, m}, v^{i, m}, t\right)=\left\{\begin{array}{cc}
1, & \text { EV } i \text { available at } t \\
0, & \text { otherwise }
\end{array}\right.
$$
make the modeling tractable.

\subsection{EV model} defined as

For each $\forall i \in \boldsymbol{N} \backslash\{1\}, \forall m \in \boldsymbol{M}_{e}^{i}$, the energy constraints, we have:
In this section, we are going to develop the modeling of EVs. The stochastic nature of EVs is first captured with uncertainty variables to show several sources that introduce randomness. It is followed by a model-free method that approximate any probability density distribution. Finally, SAA method to

One major difference between EV and BESS is the uncertainties in EV's availability and energy demand. An EV owner may arrive at parking facility at random time. Similarly, very likely the owner will not be committed to any schedule without additional incentives, therefore may leave at random time. In addition to the uncertain in availability, the energy demand of an EV highly depends on the travel distance of the owner. All these factors add up to the difficulty of DSM with EV integrations. Let $P_{e}^{i, m}$ and $\overline{P_{e}^{i, m}}$ be the maximum discharge power and charging power of EV $i$ under bus $m$, with $\underline{P_{e}^{i, m}} \in \mathfrak{R}^{1-}$ representing the effect of $\mathrm{V} 2 \mathrm{G}$. For each $\forall i \in \boldsymbol{N} \backslash\{1\}, \forall m \in \boldsymbol{M}_{e}^{i}$, we have power constrains:

$$
\underline{P_{e}^{i, m}} A_{e}^{i, m}\left(\delta^{i, m}, v^{i, m}, t\right) \leq P_{e}^{i, m}(t) \leq \overline{P_{e}^{i, m}} A_{e}^{i, m}\left(\delta^{i, m}, v^{i, m}, t\right)
$$

where $A_{e}^{i, m}\left(\delta^{i, m}, v^{i, m}, t\right) \in \mathfrak{R}^{1}$ stands for the availability of EV $i$ under bus $m$ at time $t . \delta^{i, m}$ and $v^{i, m}$ stand for the uncertainty in EV availability. The reactive power of EV bidirectional charging is not considered. The reasons behind this modeling is, in practice, EV bidirectional chargers usually has much smaller power ratings compared to BESS, making the converter more economic at the price of twoquadrant controllability [36]. So we modeled it as a constant power factor load. $A_{e}^{i, m}\left(\delta^{i, m}, v^{i, m}, t\right)$ is

$$
\underline{E_{e}^{i, m}} \leq E S_{e, t_{s}}^{i, m}\left(\chi^{i, m}\right)+\sum_{t=t_{a}^{i, m}}^{t \in \boldsymbol{T}_{i, m}} A_{\mathrm{e}}^{i, m}\left(\delta^{i, m}, v^{i, m}, t\right) P_{e}^{i, m}(t) \leq \overline{E_{e}^{i, m}}
$$


$211 E_{e}^{i, m}$ and $\overline{E_{e}^{i, m}}$ are the battery capacity limits of the EVs. $t_{a}^{i, m}$ denotes the arrival time of EV $m$ and $\boldsymbol{T}_{i, m}$ is

212 the set of time slots that EV $m$ is available. The initial SoE is represented as $E S_{e}^{i, m} \cdot \chi^{i, m}$ is used to show

213 the uncertainty in initial SoE of the EV. Similar to BESS modeling $E_{e}^{i, m}$ is set to prevent deep discharge of

214 the battery.

215 Finally, we will have the energy constraints after the whole day's operation valid for $\forall i \in \boldsymbol{N} \backslash\{1\}, \forall m \in$ $216 \boldsymbol{M}_{e}^{i}$ as:

$$
E S_{e}^{i, m}\left(\chi^{i, m}\right)+\sum_{t=t_{l}^{i, m}} A_{\mathrm{e}}^{i, m}\left(\delta^{i, m}, v^{i, m}, t\right) P_{e}^{i, m}(t)=E F_{e}^{i, m}\left(\chi^{i, m}\right)
$$

$218 E F_{e}^{i, m}$ stands for the final SoE of the EV and $t=t_{l}^{i, m}$ stands for the leave time of EV $m$. It is clear that for 219 an arbitrary distribution of random variables $\delta^{i, m}, v^{i, m}, \chi^{i, m}$, it is difficult to represent Eqn.(5.e)-(5.f), 220 (6.a)-(6.d) analytically, making even more drag when solving the entire DSM. Let Eqn.(5.e)-(5.f), 221 Eqn.(6.a)-(6.d) be original problem, described by random variables $\delta^{i, m}, v^{i, m}$ and $\chi^{i, m}$, we need to 222 develop a numerical method that can capture the randomness and closely approaches the original 223 problem.

\section{3.2. Model-free Probability Density Estimation}

225 In the previous subsection, we model the EV with stochastic variables $\delta^{i, m}, v^{i, m}, \chi^{i, m}$, showing the 226 sources of uncertainties in EVs. This subsection presents the method to model the distribution of 227 stochastic variables, which will be used in numerical approximations in the following section. The 228 method studied in this subsection can be applied to EVs with different arrival time, departure time and energy demands.

230 Given an empirical realization of certain stochastic variable, here we present Kernel Density Estimation $231(\mathrm{KDE})$ to capture the empirical probability density. The mathematical representation of KDE is described 232 as follows:

$$
\rho(x)=\frac{1}{N} \sum_{i=1}^{N} K\left(\frac{x-x_{i}}{b}\right)
$$


where $N$ is the number of kernels used, $x_{i}$ is the center of one kernel, $b$ denotes the bandwidth of the

235 kernel, $K(\cdot)$ is the kernel function, and $\rho(\cdot)$ is the probability density function. I

236 Empirical probability density is usually given in histogram. The center of the each kernel naturally falls 237 into the center of each bin. The number of kernels is equal to the number of bins in the histogram. The 238 bandwidth then becomes the only free parameter to be determined. A common way to determine the 239 bandwidth is to use the rule-of-thumb principle described in [38]. After determining each parameters, the discrete histogram can be modeled by continuous KDE. This KDE is then utilize for scenario generation in the following section.

\subsection{Sample Average Approximation}

243 To ensure the stochastic modeling of EVs can be widely adopted without the constraints for certain 244 known standard distributions (such as Gaussian and uniform distribution). To this end, we use a 245 distribution-free numerical method SAA to reformulate the problem in a tractable manner. Literatures have shown that if the optimization problem involves continuous decision variables, and the random variables are i.i.d.; then by generating scenarios from the distribution of random variables, the average of these generated scenarios approaches the expectation as the number of scenario grows [39]. In this paper, the decision variables are not integers and therefore obviously continuous. An efficient approximation of Eqn.(5.e) and (5.f) are offered by generating NS i.i.d. samples of

$251 \quad \boldsymbol{\psi}=\left\{\delta_{s}^{i, m}, v_{s}^{i, m}, \chi_{s}^{i, m}\right\}_{s=1}^{N S}$ :

$$
P^{i}(t)=P_{l}^{i}(t)+\sum_{m \in M_{b}^{i}} P_{b}^{i, m}(t)+\frac{1}{N S} \sum_{S \in \boldsymbol{\psi}} \sum_{m \in \boldsymbol{M}_{e}^{i}} P_{e, s}^{i, m}(t)
$$

$$
Q^{i}(t)=Q_{l}^{i}(t)+\sum_{m \in M_{b}^{i}} Q_{b}^{i, m}(t)+\frac{1}{N S} \sum_{s \in \boldsymbol{\psi}} \sum_{m \in M_{e}^{i}} Q_{e, s}^{i, m}(t)
$$

254 where we use a subscript $s$ for each random variable to denote the generated scenario. The assumption 255 here is $\delta_{s}^{i, m}, v_{s}^{i, m}, \chi_{s}^{i, m}$ are i.i.d, we will justify this assumption in the studied case Section 5. Accordingly, 256 for each generated scenario $s \in \boldsymbol{\psi}$, the constraints for EV should still hold for each generated scenario.

257 Therefore, Eqn.(6.a)-(6.d) are updated as the following:

$$
\underline{P_{e}^{i, m}} A_{e, s}^{i, m}(t) \leq P_{e, s}^{i, m}(t) \leq \overline{P_{e}^{i, m}} A_{e, s}^{i, m}(t)
$$




$$
A_{e, s}^{i, m}(t)=\left\{\begin{array}{cc}
1, & \text { EV } i \text { available at } t \text { in } s \\
0, & \text { otherwise }
\end{array}\right.
$$

$$
\underline{E_{e}^{i, m}} \leq E S_{e, s}^{i, m}+\sum_{t=t_{a, s}^{i, m}}^{t \in T_{i, m}^{s}} A_{\mathrm{e}, \mathrm{s}}^{i, m}(t) P_{e, s}^{i, m}(t) \leq \overline{E_{e}^{i, m}}
$$

$$
E S_{e, s}^{i, m}+\sum_{t=t_{l, s}^{i, m}} A_{\mathrm{e}}^{i, m}(t) P_{e}^{i, m}(t)=E F_{e, s}^{i, m}
$$

262 where following the naming convention, $t_{a, s}^{i, m}$ and $t_{l, s}^{i, m}$ respectively stands for the arrival and leave time 263 of EV $m$ in scenario s. Similarly, $\boldsymbol{T}_{i, m}^{S}$ is the set of time slots that EV $m$ is available in scenario $s$. Note the 264 above constraints hold for $\forall s \in \boldsymbol{\psi}, \forall i \in \boldsymbol{N} \backslash\{1\}, \forall m \in \boldsymbol{M}_{e}^{i}$. We also describe how to capture the 265 distribution of the uncertainty variables, i.e. $\delta^{i, m}, v^{i, m}, \chi^{i, m}$, in Section 5. After the SAA numerical 266 approximation, the optimization problem is tractable. We define the decision variable set to be $267 \boldsymbol{\Omega}=\left\{P^{i}(t), Q^{i}(t), P_{b}^{i, m}(t), Q_{b}^{i, m}(t), P_{e, s}^{i, m}(t), Q_{e, s}^{i, m}(t) \mid t \in \boldsymbol{T}\right\}$. The DSM problem now can be presented as:

$$
\begin{gathered}
\min _{\Omega} \sum_{t} \Pi(\mathrm{t}) \sum_{i \in N \backslash\{1\}} P^{i}(t)+\gamma \sum_{t} \sum_{(i, j) \in \xi} R_{i j}\left|i_{i j}(t)\right|^{2} \\
\text { subject to. (2), (4), (5.a)-(5.d), (8), (9) }
\end{gathered}
$$

268 However, Eqn.(4.d) is non-convex, making the problem impossible to solve with convex optimization 269 solvers. Non-convex solvers require much stronger computational power and the solutions are not 270 guaranteed to be global optimum. As a result, we seek to convexify the DSM problem and solve it in a 271 distributed manner using ADMM in the following section.

\section{Distributed DSM}

273 In this paper, we target to develop a scalable and privacy-preserving DSM. To this end, we use a 274 distributed algorithm ADMM to distribute centralized computational burden of DSO to each SDSO. The 275 distributed algorithm also protects the end-user's privacy by getting only the "aggregated" information 
under a bus. To guarantee convergence, we relax the original non-convex problem and show conditions

277 for a tight relaxation.

278

279

280

281

282

283

284

285

286

287

288

289

290

291

292

293

\subsection{Convex Relaxation}

According to [31] and [32], the non-convexity constraint in Eqn.(4.d) can be relaxed to a convex secondorder cone as follows:

$$
\left|v_{i}(t)\right|^{2}\left|i_{i j}(t)\right|^{2} \geq P_{i j}^{2}(t)+Q_{i j}^{2}(t), \forall(i, j) \in \xi, j \neq 1
$$

For a tight relaxation, there are three sufficient conditions, namely (a) the network has to be radial; (b) the power injection to each bus cannot be too large; and (c) bus voltages are kept around nominal values. The method to check the exactness of a relaxation is to solve for Eqn.(10) and compare the lefthand-side in the equality to the right-hand-side. We define the decision variable set to be $\boldsymbol{\Omega}=$ $\left\{P^{i}(t), Q^{i}(t), P_{b}^{i, m}(t), Q_{b}^{i, m}(t), P_{e, s}^{i, m}(t), Q_{e, s}^{i, m}(t) \mid t \in \boldsymbol{T}\right\}$. With the convex relaxation, the relaxed DSM can be described as follows:

$$
\begin{gathered}
\min _{\Omega} \sum_{t} \Pi(\mathrm{t}) \sum_{i \in N \backslash\{1\}} P^{i}(t)+\gamma \sum_{t} \sum_{(i, j) \in \xi} R_{i j}\left|i_{i j}(t)\right|^{2} \\
\text { subject to (2), (4.a)-(4.c),(5.a)-(5.d), (8) - (10) }
\end{gathered}
$$

Given a tight convex relaxation, the DSM is in a centralized manner and therefore the DSO still has to collect the information on each buses, raising privacy concerns to EV owners. Furthermore, with a growing size of BESSs, EVs and perhaps other controllable devices not included in this paper, the DSO has a heavy computational burden for the system to scale up. These motivates us to develop a distributed DSM that is scalable and privacy-preserving.

\subsection{Distributed Algorithm}

We develop the distributed DSM using the ADMM method (see Appendix for details). The key for ADMM is for a convex problem can be decomposed into global and local sub-problems. The ADMM method actually turns out to be natural fit to DSM problem in this paper. We decompose the original DSM problem into a global problem that solves for the OPF and VR at DSO, and local problems that solve for energy cost minimization at SDSOs.

Before start of the ADMM iterations, we first initialize the problem with random numbers: for the first iteration, DSO sets random values to $P^{i}$ and $Q^{i}$, for $\forall i \in N \backslash\{1\}$. For simplicity of notation, we neglect 
301

302

303

304

305

306

$$
\begin{aligned}
\min _{\boldsymbol{\Omega}_{i}} \sum_{t} \Pi(t) P^{i}(t) & +\frac{\rho_{1}}{2} \sum_{t}\left(-\left(P^{i}(t)\right)_{k}+P_{l}^{i}(t)+\sum_{m \in \boldsymbol{M}_{b}^{i}} P_{b}^{i, m}(t)+\frac{1}{N S} \sum_{s \in \boldsymbol{\psi}} \sum_{m \in \boldsymbol{M}_{e}^{i}} P_{e, s}^{i, m}(t)+\left(\alpha_{i}(t)\right)_{k}\right)^{2} \\
+ & \frac{\rho_{2}}{2} \sum_{t}\left(-\left(Q^{i}(t)\right)_{k}+Q_{l}^{i}(t)+\sum_{m \in \boldsymbol{M}_{b}^{i}} Q_{b}^{i, m}(t)+\frac{1}{N S} \sum_{s \in \boldsymbol{\psi}} \sum_{m \in \boldsymbol{M}_{e}^{i}} Q_{e, s}^{i, m}(t)+\left(\beta_{i}(t)\right)_{k}\right)^{2}
\end{aligned}
$$

subject to (2), (9)

After receiving all the information for iteration $k+1$ from SDSO, DSO solve a global DSM problem by every time step as follows:

$$
\begin{gathered}
\min _{P^{i}(t), Q^{i}(t)} \gamma \sum_{(i, j) \in \xi} R_{i j}\left|i_{i j}(t)\right|^{2}+\frac{\rho_{1}}{2} \sum_{i \in N \backslash\{1\}}\left(-P^{i}(t)+\left(P_{a}^{i}(t)\right)_{k+1}+\left(\alpha_{i}(t)\right)_{k}\right)^{2} \\
+\frac{\rho_{2}}{2} \sum_{i \in N \backslash\{1\}}\left(-Q^{i}(t)+\left(Q_{a}^{i}(t)\right)_{k+1}+\left(\beta_{i}(t)\right)_{k}\right)^{2}
\end{gathered}
$$

subject to (4.a) - (4.c), (5.a) - (5.d), (10)

where $\rho_{1}, \rho_{2}$ are the step size. The local DSM at each bus solve for the optimal schedule for BESS and EV in iteration $k+1$, denoted as $\left(P_{b}^{i, m}\right)_{k+1},\left(P_{e, s}^{i, m}\right)_{k+1},\left(Q_{b}^{i, m}\right)_{k+1}$ and $\left(Q_{e, s}^{i, m}\right)_{k+1}$. Then the SDSO communicate the aggregated bus load $\left(P_{a}^{i}\right)_{k+1}$ and $\left(Q_{a}^{i}\right)_{k+1}$ defined as follows to DSO.:

$$
\begin{aligned}
& \left(P_{a}^{i}\right)_{k+1}=P_{l}^{i}(t)+\sum_{m \in \boldsymbol{M}_{b}^{i}}\left(P_{b}^{i, m}(t)\right)_{k+1}+\frac{1}{N S} \sum_{s \in \boldsymbol{\psi}} \sum_{m \in \boldsymbol{M}_{e}^{i}}\left(P_{e, s}^{i, m}(t)\right)_{k+1} \\
& \left(Q_{a}^{i}\right)_{k+1}=Q_{l}^{i}(t)+\sum_{m \in \boldsymbol{M}_{b}^{i}}\left(Q_{b}^{i, m}(t)\right)_{k+1}+\frac{1}{N S} \sum_{s \in \boldsymbol{\psi}} \sum_{m \in \boldsymbol{M}_{e}^{i}}\left(Q_{e, s}^{i, m}(t)\right)_{k+1}
\end{aligned}
$$$$
\text { subject to }(4 . a)-(4 . c),(5 . a)-(5 . d),(10)
$$ 
314 After solving for each time step of a day, the global DSM, the DSO updates the two sets of dual variables

315 for each bus $i \in N \backslash\{1\}$ as follows:

$$
\begin{aligned}
& \left(\alpha_{i}(t)\right)_{k+1}=\left(\alpha_{i}(t)\right)_{k}-\left(P^{i}(t)\right)_{k+1}+\left(P_{a}^{i}(t)\right)_{k+1} \\
& \left(\beta_{i}(t)\right)_{k+1}=\left(\beta_{i}(t)\right)_{k}-\left(Q^{i}(t)\right)_{k+1}+\left(Q_{a}^{i}(t)\right)_{k+1}
\end{aligned}
$$

317 The updates of the dual variables signals the end of iteration $k+1$. The updated $\left(P^{i}\right)_{k+1},\left(Q^{i}\right)_{k+1}$, $318\left(\alpha_{i}\right)_{k+1}$ and $\left(\beta_{i}\right)_{k+1}$ are broadcasted to each SDSO for the next iteration. The iteration ends when the 319 problem converges (discussed in Section 4.3). The ADMM based algorithm can distribute the 320 computational burden at the DSO to SDSO. At the same time, for each iteration, the DSO only has to 321 know the aggregated load at each bus, preserving the privacy by hiding the individual information in the 322 aggregated information.

\subsection{Convergence and Over Relaxation}

324 It has been proved in [28] that if the centralized problem is convex, the ADMM is to converge to the 325 same optimum with tolerable errors. For the proposed ADMM based DSM, the stopping criteria is when 326 the distributed algorithm converges. Define the primal residuals of the two dual variables at iteration $k$ 327 for each bus $i$ to be:

$$
\begin{aligned}
& r_{p}^{i}=\sum_{t=H}\left(-\left(P^{i}(t)\right)_{k}+\left(P_{a}^{i}(t)\right)_{k}\right)^{2} \\
& r_{q}^{i}=\sum_{t=H}\left(-\left(Q^{i}(t)\right)_{k}+\left(Q_{a}^{i}(t)\right)_{k}\right)^{2}
\end{aligned}
$$

329 The stopping criteria for the distributed DSM is to make sure the two primal residuals to be sufficiently 330 small:

$$
r_{p}^{i} \leq \epsilon, r_{q}^{i} \leq \epsilon, \forall i \in N \backslash\{1\}
$$

332 where $\epsilon$ is a predefined error boundary. The summary of the ADMM based distributed DSM is tabulated 333 in Algorithm 1.

Algorithm 1. ADMM based distributed DSM 
1: Initialize the problem. Set $k=1$. DSO randomly choose $P^{i}, Q^{i}, \alpha_{i}$ and $\beta_{i}{ }^{*}$. SDSO at bus $i$ randomly choose $P_{b}^{m, i}, Q_{b}^{m, i}$, and $P_{e, s}^{m, i}$ and $Q_{e, s}^{m, i}$.

2: $\quad$ Repeat for $k=2,3, \ldots$

3: $\quad$ Solve the local DSM in (11) at each SDSO using $\left(P^{i}\right)_{k},\left(Q^{i}\right)_{k},\left(\alpha_{i}\right)_{k}$ and $\left(\beta_{i}\right)_{k}$ broadcasted by DSO

4: $\quad$ Solve the global DSM in (12) at DSO by collecting $\left(P_{a}^{i}\right)_{k+1}$ and $\left(Q_{a}^{i}\right)_{k+1}$ from each SDSO

5: $\quad$ Update dual variables in (13) and broadcast $\left(P^{i}\right)_{k+1},\left(Q^{i}\right)_{k+1},\left(\alpha_{i}\right)_{k+1}$ and $\left(\beta_{i}\right)_{k+1}$

6: Until convergence in (14)

* for simplicity of notation, time $t$ is neglected for each variable

To accelerate the convergence speed, an over relaxation technique is proposed in [38]. Basically, it is to substitute the $\left(P_{a}^{i}\right)_{k+1}$ and $\left(Q_{a}^{i}\right)_{k+1}$ in each iteration $k+1$ (line 5 in Algorithm 1) as follows:

$$
\begin{aligned}
& \left(P_{a}^{i}(t)\right)_{k+1}=\theta\left(P_{a}^{i}(t)\right)_{k+1}-(1-\theta)\left(-\left(P^{i}(t)\right)_{k}\right) \\
& \left(Q_{a}^{i}(t)\right)_{k+1}=\theta\left(Q_{a}^{i}(t)\right)_{k+1}-(1-\theta)\left(-\left(Q^{i}(t)\right)_{k}\right)
\end{aligned}
$$

where $\theta$ is a relaxation parameter. [38] suggested that $\theta \in[1.5,1.8]$ can improve the convergence speed. We will provide numerical verifications and comparisons to the original ADMM based DSM in the following section.

\section{Numerical Results and Analysis}

This section demonstrates the correctness of the proposed DSM and its performance. We first introduce the simulation setup. It is followed by case studies to verify the correctness, and extensive discussions on understanding the numerical results.

\subsection{Simulation Setup}

We use a modified IEEE 13-bus radial test feeders [40] for demonstration of the proposed DSM. As the primary focus of this paper is not on dynamics or electromagnetic transient, we made some minor modification to the original IEEE 13-bus radial test feeders to study the steady-state DSM: the in-line transformer between bus 633 and 634 is removed; the breaker between bus 671 and 692 is closed. Because of the extreme short line length between bus 633 and 634, the two buses are treated as one 
single bus, i.e. bus 5 in Fig.3. For similar reason, bus 692 and 675 are treated as bus 9 in Fig.3. The root

352 node is a $115 \mathrm{kV}$ substation and it is connected with a $115 \mathrm{kV} / 4.16 \mathrm{kV}$ transformer. We do not consider

353 the three-phase imbalance in the distribution system, the three-phase diagram is therefore drawn as a

354 one-line diagram. At each distribution bus, it is connected to several $4.16 \mathrm{kV} / 0.12 \mathrm{kV}$ distribution 355 transformers. The transformers are directly connected to the load, BESSs and EVs.

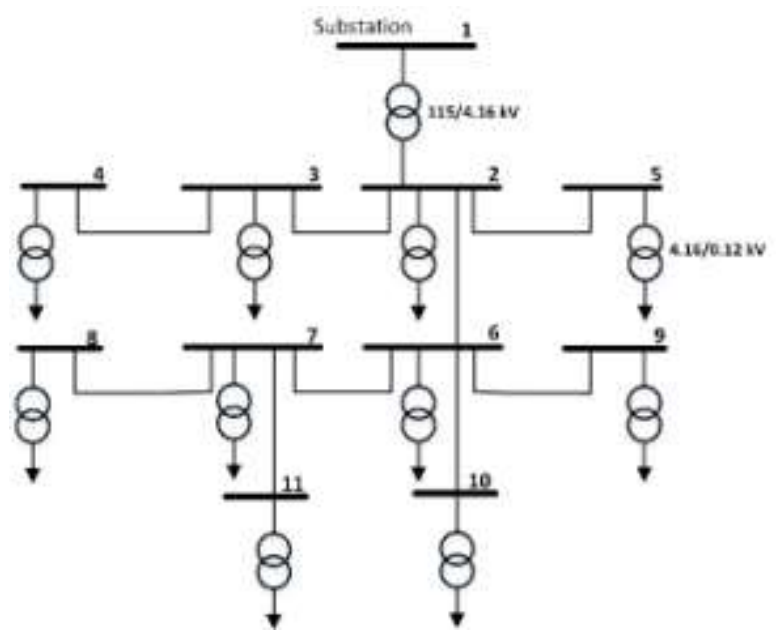

Fig.3. Modified IEEE 13-bus radial test feeders

The DSM studied in this paper uses one hour as time step. We use the day-ahead price from [40], which is shown in Fig.4. Fixed load profile at each bus is obtained from [42] and plotted in Fig.5. We place one BESS at each buses in Fig.3 except for the root bus. The key parameters of three studied types of batteries are documented in Table 1.

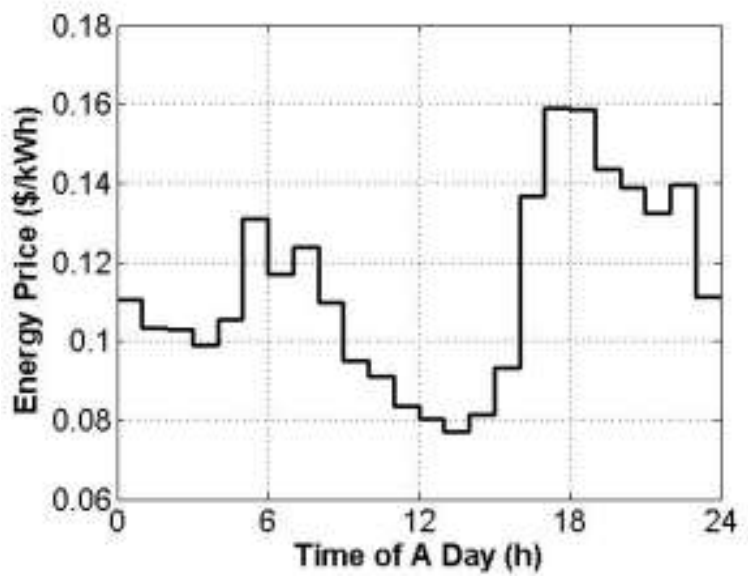

Fig.4. Day-ahead price 


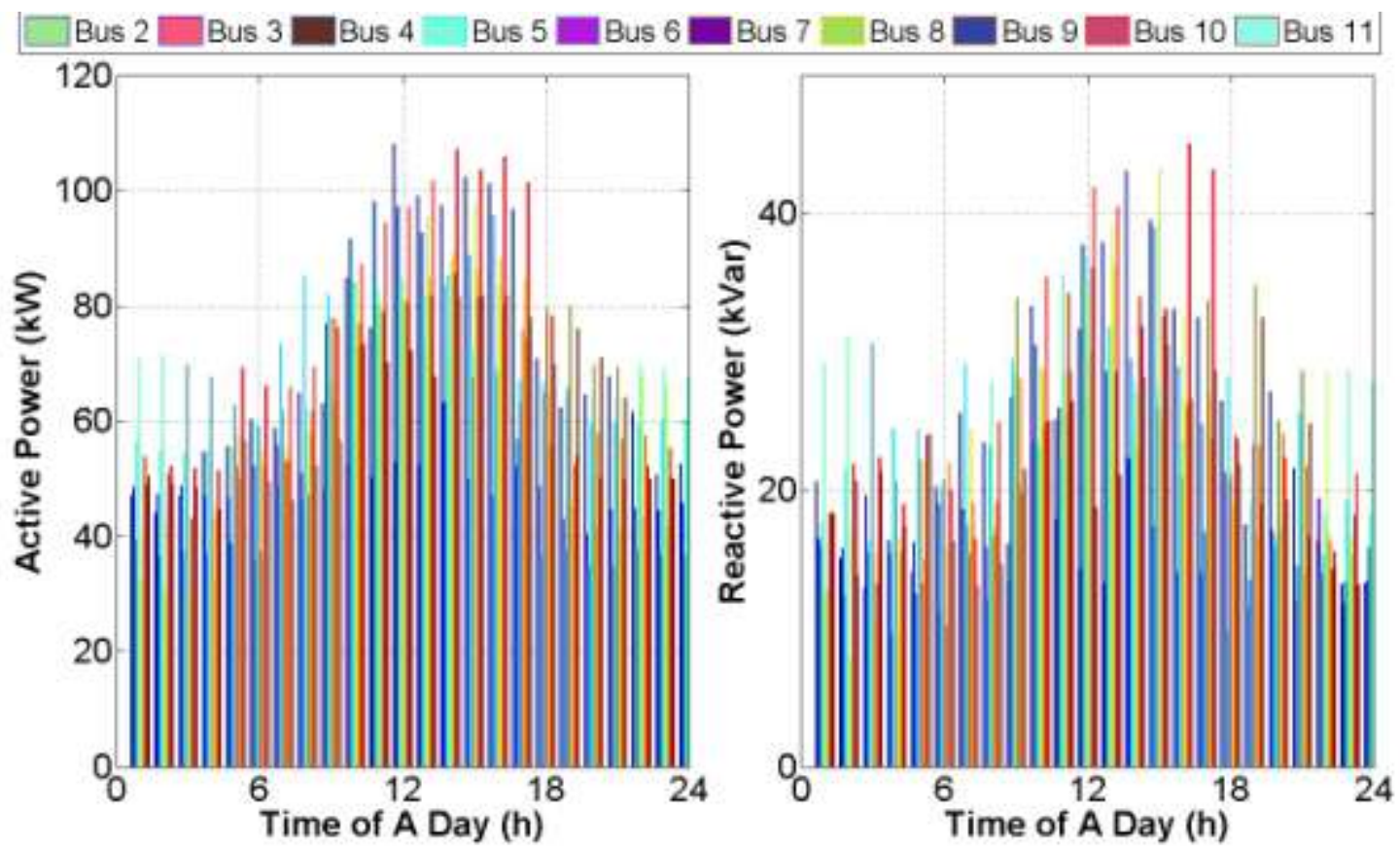

Fig.5. A typical day load pattern

366 We collected 19 EV driver's records for a whole year on UCLA campus in order to accomplish this study.

367 The recorded EVs fall into three popular models in the market, i.e. Nissan Leaf, Tesla Model S and 368 Mitsubishi MiEV. Their arrival time, leaving time, and energy demands are recorded for generating a 369 close to real-life study. Apart from filtering out data with communication failures, we made the 370 following assumptions to the original data in order to verify the proposed DSM: 1) V2G capability. Very 371 few charging stations supports V2G nowadays, partly resulting from different interfacing protocols, see 372 two different protocols used in the three mentioned EV models in [43] and [44]. The records we have 373 collected are EV charging data, however, it reveals the V2G potential: An EV is usually fully charged 374 before it is unplugged and therefore can perform V2G in spare time. Following this reasoning, we 375 assume EVs are V2G capable once connected to the grid, however they need to meet their energy 376 demands before leave. 2) SoE acquisition. The SoE data is also hard to obtain and therefore it is 377 impossible to tell the starting SoE after EV connects. As we have data in EV energy demands, we will 378 assume EVs get fully charged every time. In this way, it will be tractable to calculate the starting SoE 379 using the collected energy demands data. Table 1 summarizes the key parameters used for three 380 different EV models and BESS models in this paper. Table 2 summarizes the key parameters used for 381 distribution network simulation. All EV related parameters comes from real-life EV manufacturers. BESS 382 related parameters are chosen as these are the typical size of the BESS in distribution systems. And 
finally, distribution network related parameters are from IEEE benchmark test system and real-life 384 regulations in U.S power systems.

Table 1. Key Parameters Used for BESS and EV

\begin{tabular}{lcc}
\hline \multicolumn{1}{c}{ Device } & Power Rating (Min, Max) & Capacity \\
\hline Type A BESS & {$[-20,20] \mathrm{kVA}$} & $25 \mathrm{kWh}$ \\
Type B BESS & {$[-15,15] \mathrm{kVA}$} & $20 \mathrm{kWh}$ \\
Type C BESS & {$[-10,10] \mathrm{kVA}$} & $15 \mathrm{kWh}$ \\
Tesla Model S & {$[-3.3,6.6] \mathrm{kW}$} & $65 \mathrm{kWh}$ \\
Nissan Leaf & {$[-3.3,6.6] \mathrm{kW}$} & $24 \mathrm{kWh}$ \\
Mitsubishi MiEV & {$[-3.3,6.6] \mathrm{kW}$} & $16 \mathrm{kWh}$ \\
\hline
\end{tabular}

386

387

Table 2. Key Parameters Used for Distribution Networks

\begin{tabular}{lc}
\hline \multicolumn{1}{c}{ Parameters } & Rating \\
\hline Voltage Magnitude Limit & {$[0.95$ 1.05] p.u. } \\
Substation Power Limit & $5 \mathrm{MVA}$ \\
Substation Secondary Voltage & $4.16 \mathrm{kV}$
\end{tabular}

388

389 In observance of the large number of EV owners, we only show three EV owner profiles in Fig. 6 as the 390 representatives of the 19 EV owners. The three owners drive a Tesla Model S, Nissan Leaf and Mitsubishi 391 MiEV respectively. We capture the distribution of the real-life data using KDE described in section 3.2. 392 Fig.6 compares the real-life data and distribution generated by KDE, showing a close match between the 393 two. The KDE is then applied to generate the scenarios in SAA. We duplicate 1 out of the 19 EVs to make 394 the total EV number 20, and we evenly distribute the two EVs at each distribution bus except for the 395 root node. For the distributed DSM, We set weighting factor $\gamma=1$, step size $\rho_{1}=1, \rho_{2}=2$ and 396 predefined error boundary $\epsilon=10^{-3}$. The over relaxation parameter is set to $\theta=1.75$. The proposed 397 DSMs are solved with Gurobi [45] on a PC with 2.67GHz CPU and 8 GB RAM. 

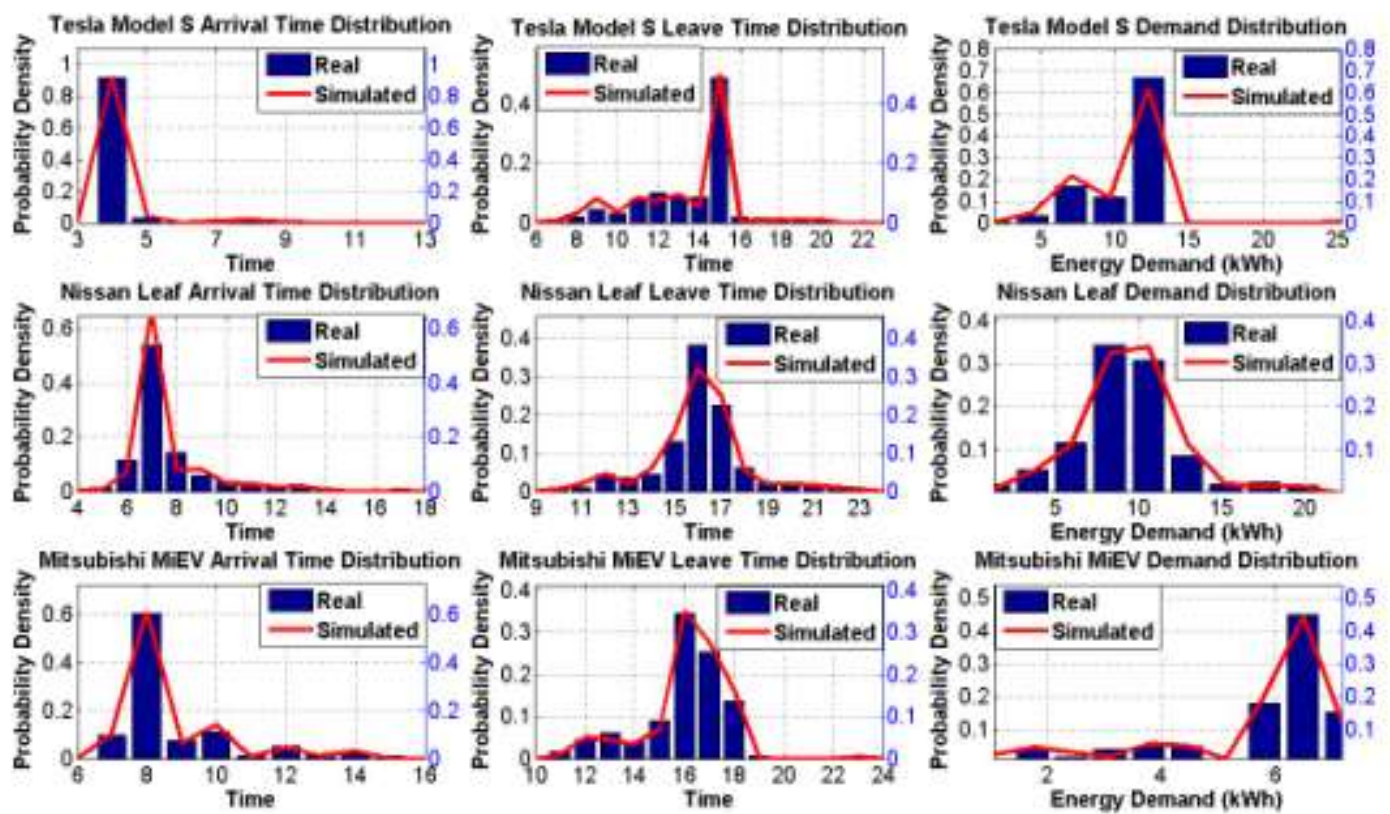

Fig.6. Typical patterns of EV users

400

401

\subsection{Case Study}

We generate 200 scenarios using the SAA and the convergence of the proposed DSM is presented in Fig. 7. Fig.7(a) shows the convergence of objective function for both over-relaxed ADMM (o-ADMM) DSM and regular ADMM DSM. Both DSMs converges to the optimum solved in a centralized manner. After the DSMs converge, the performance of both DSMs are stable. Furthermore, we pick the slowest converging bus, bus 11, to take a closer look at the convergence defined by Eqn.(14) in Fig.7(b). It shows the primal dual residuals of the o-ADMM DSM and ADMM DSM at bus 11 for both active (P) and reactive power (Q) corresponding to Eqn.(14). It is observed that the over relaxation speeds up the convergence: the o-ADMM DSM converges in 36 iterations while the ADMM DSM takes 70. After 80 iterations, the o-ADMM DSM hits its performance limitation: the residuals stop to decline and stay in an acceptable range. Moreover, we keep tracking the convex relaxation in Eqn.(10) and found it to be tight for all iterations. In a word, the o-ADMM based DSM converges relatively fast.

In the proposed DSM, SAA numerically approaches the stochastic optimization problem. To further validate the performance of SAA, we vary the size of the generated scenario size to examine the impact of scenario size on the DSM. As shown in Table 3, we vary the scenario size for 100, 200 and 300, and run the numerical experiment using the same setting for 10 times. We obtain the mean and standard deviation of the 10 experiments for each scenario size. Comparing the results of different scenario size, 
417 the mean of the three sets of experiments are close. As expected, the 300 set has a smaller standard 418 deviation. The scenarios size is directly related to the size of the optimization problem, and a large 419 scenario size requires a lot of computational resources. As a conclusion, using 200 scenarios is a safe 420 approach to approximate the EV randomness in the studied setting. For cases requiring medium 421 accuracy, 100 generated scenarios can satisfy the requirements.
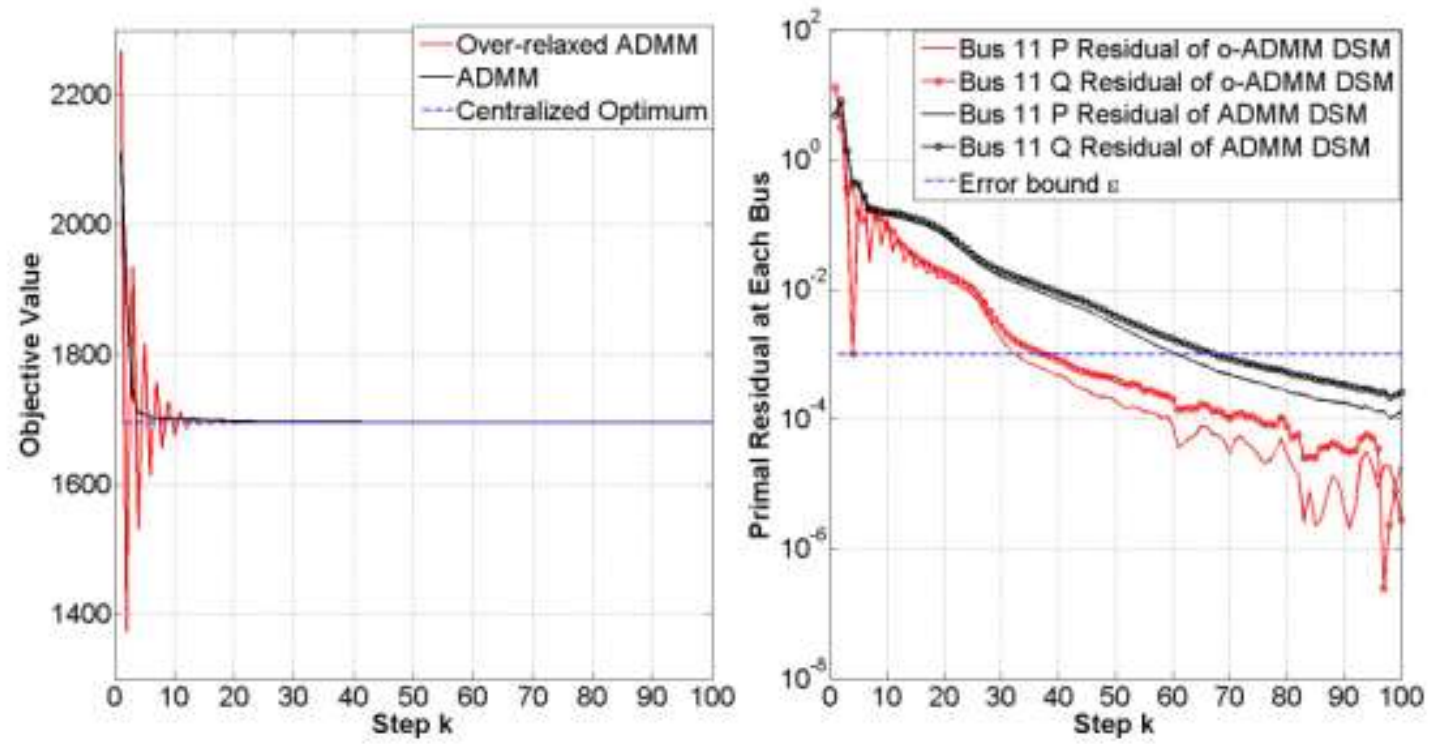

Fig.7. (a) Convergence of the Distributed DSMs and (b) primal residuals of bus 11 Table3. Mean and Standard Deviation of Objective Value vs. Scenario Size

\begin{tabular}{c|ccc} 
Scenarios & 100 & 200 & 300 \\
\hline Mean & 1695.33 & 1695.29 & 1696.32 \\
STD & 0.1522 & 0.1199 & 0.0892
\end{tabular}

425 To demonstrate the correctness of the numerical experiments, we also present load profile under bus

426 11. Bus 11 is picked for analysis as it is the bus most distanced from the root node. As voltage drop is 427 most significant for the buses far away from the root node, we would like to make sure all the 428 constraints are met at bus 11 . Firstly, we examined the load profile under bus 11, the battery's active and reactive power together with two EV profiles are shown in Fig.8.(a). Note that the EV profile is one generated scenario out of 200 total scenarios. It is shown that the battery is most of the time supporting reactive power, which is expected for compensate for the voltage drop in distribution system. 432 Furthermore, in Fig.8.(b), we align the time axis and present the State-of-Charges (SoCs) for batteries 
and EVs in the same scenario. For easy representation, we normalized the SoE used in Section 3 to SoC

434 in figures. By comparing Fig.8.(a) and Fig.8.(b), it is clearly shown that the EV1 arrives at 5am and leave

(a)

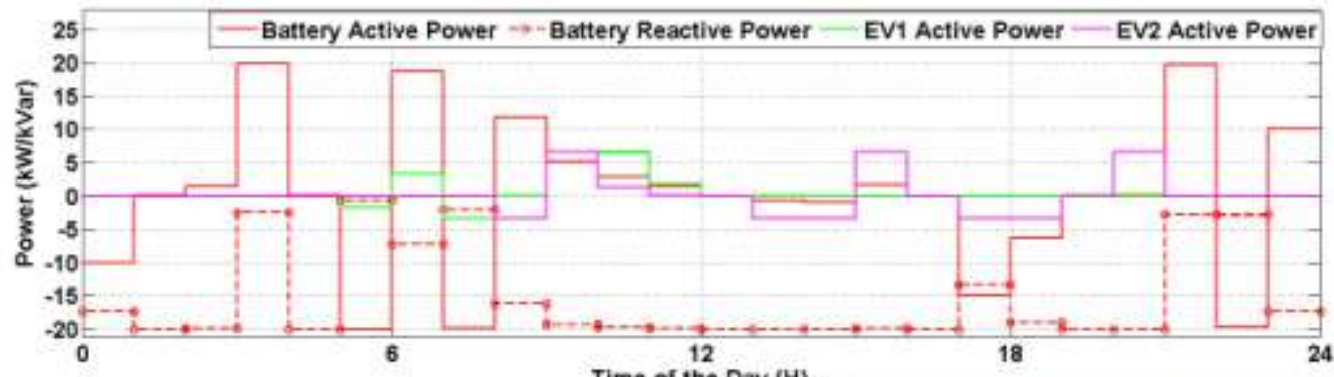

(b)

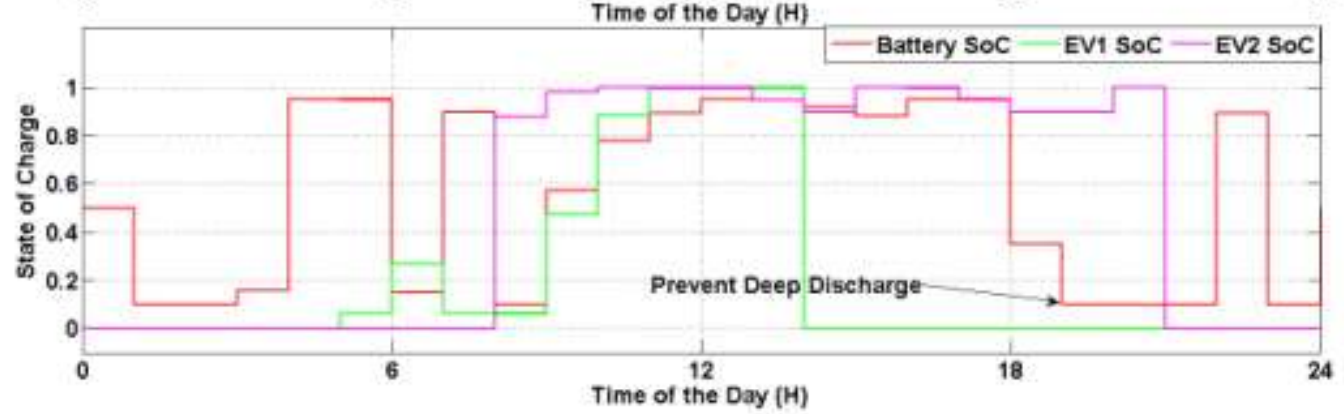

Fig.8. SDSO accessible: (a) Load profile under bus 11, (b) SoC of batteries and EVs

441 Besides checking the load profiles and SoCs, we further presents the bus aggregated load and voltage

442 profile from a DSO's point of view. The aggregated active load, reactive load and voltage profile for the 443 same bus (bus 11) is shown in Fig.9. We notice that the voltage regulation is performed during 10am and $4445 \mathrm{pm}$, a time window when system has heavy active load and voltage profile decays accordingly. By comparing Fig.9 with Fig.8(a), we find out that battery is under reactive power compensation mode in the same time period. Fig.8 and Fig.9 mutually verify the correctness of the proposed DSM. 


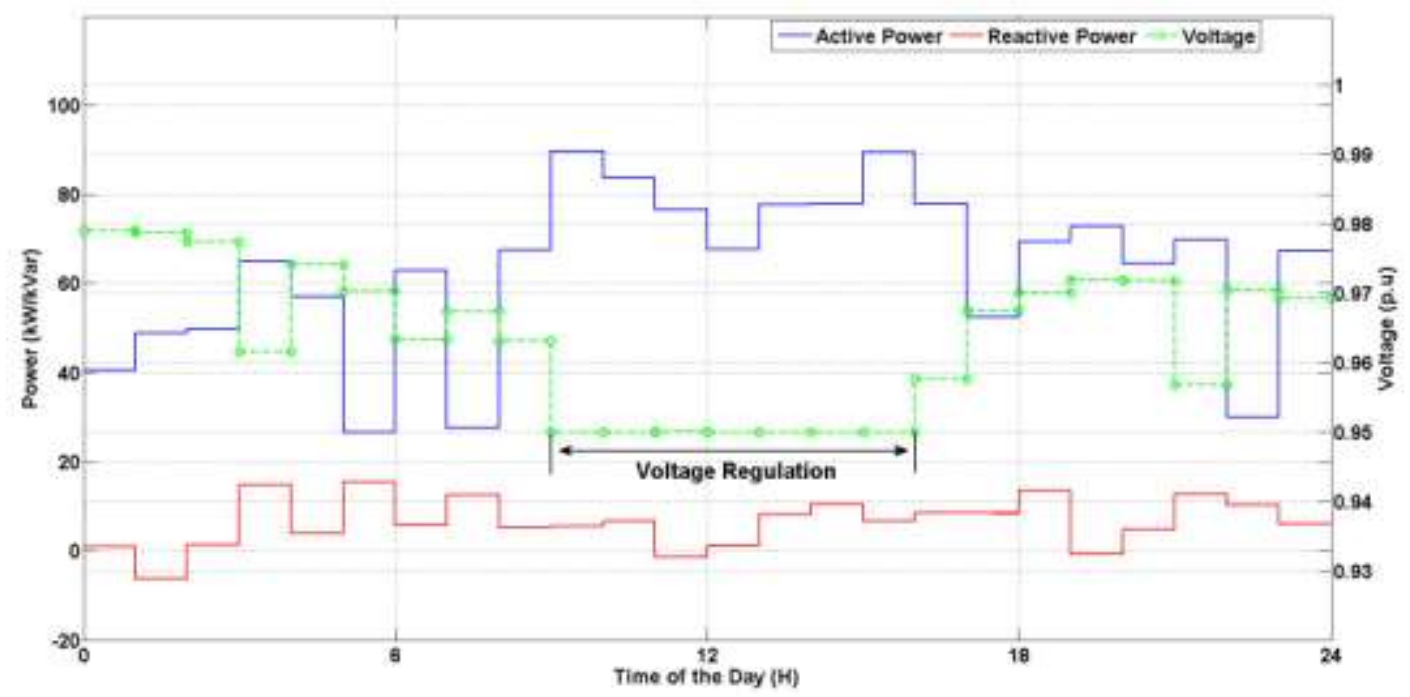

Fig.9. DSO accessible: Bus 11 aggregated load profile and bus voltage

\subsection{Discussions}

450 In this subsection, we are going to discuss three interesting observations of the proposed DSM.

451 1) Scalability. Fig.10 shows the scalability of the proposed DSM under different size of the EV number.

452 We change the number of EVs in the distribution network, from $20 \mathrm{EVs}$ in the original setting to $40 \mathrm{EVs}$ 453 and 60 EVs. Given the limitation of actual EV user profile we have collected, we duplicate the number of 454 EVs under each bus to increase the EV number. We run the numerical experiment by keeping all the 455 other settings the same. In Fig.10, it can be clearly seen that as the EV number increases, the problem 456 size scale up linearly with the EV number. However, as the stochastic optimization needs to generate a 457 large number of scenarios for each EV (in this case 200), the problem size actually grows very fast. By 458 integrating $60 \mathrm{EVs}$, it is already extremely slow to solve the DSM in a centralized way. On the other hand, 459 if we solve it in a distributed manner, the computational burdens are distributed to SDSO. In Fig.10, we 460 show three cases of numerical results, namely unstable termination, stable termination and termination 461 with reduced line length. a) the unstable termination refers to the case whose termination criteria is 462 met according to Eqn.(14) in certain iterations. However, if the algorithm continues to run for one more 463 iteration, the termination criteria is violated. A good example of this case is shown in Fig.7(b): at 464 iteration 5, the oscillation introduced by the relaxed term in Eqn.(15) has reduced the error below the 465 pre-set error bound. However, such oscillation has enlarged the error above the error bound in the next iteration. b) the stable termination stands for termination where error continues to stay below error 
bound. An illustrative example is Fig.7(b) at iteration 36. After this iteration, the error continuously stays

468 below error bound. c) we also show the scalability of the same problem with a reduced distribution line 469 length. The line length in this case is reduced to one fifth of the original length.

470 There are three observations for this numerical experiment: a) by comparing the unstable termination 471 under different size of EV integration, we see a good chance that the o-ADMM based DSM terminates in 472 10s of iterations. The number of iterations does not grow much as the problem size grows. b) by 473 comparing the stable terminations, we find it scales as the problem scales up. By fitting a first order 474 polynomial between the number of stable iterations and the problem size, it is found out that the 475 distributed method needs to increase one iteration with every increment of 2000 decision variables. 476 There is a chance where the introduced oscillation terminates the algorithm in early stage, however, 477 such termination is not guaranteed. However, in order to obtain guaranteed (stable) termination, the 478 iteration scales up with the problem size. In other words, the distributed DSM reduce the computational 479 burden for the central controller at the expense of longer computational time. c) by comparing the 480 termination with reduced line length case, we further find out that the increase in stable termination 481 actually results from a harder problem: the increase in EV numbers (and hence in energy demand) in the 482 system has made the voltage regulation constraints hard to be met. However, if we reduce the line 483 length of the distribution system, the constraints are more easily met and hence iterations do not 484 increase with the problem size.

485 If the EV size continuous to grow, it can be projected that the problem is harder to solve. With the 486 introduction of more EVs, it not only increases the number of decision variables, at the same time, the 487 EV charging demands also make the voltage and power flow constraints harder to be met. If the EV size 488 is over several hundred, the model used in this paper may need further adjustments: instead of 489 modeling the stochastic behavior of each individual EV, stochastic modeling should be performed for 490 aggregated EV load under each bus. 


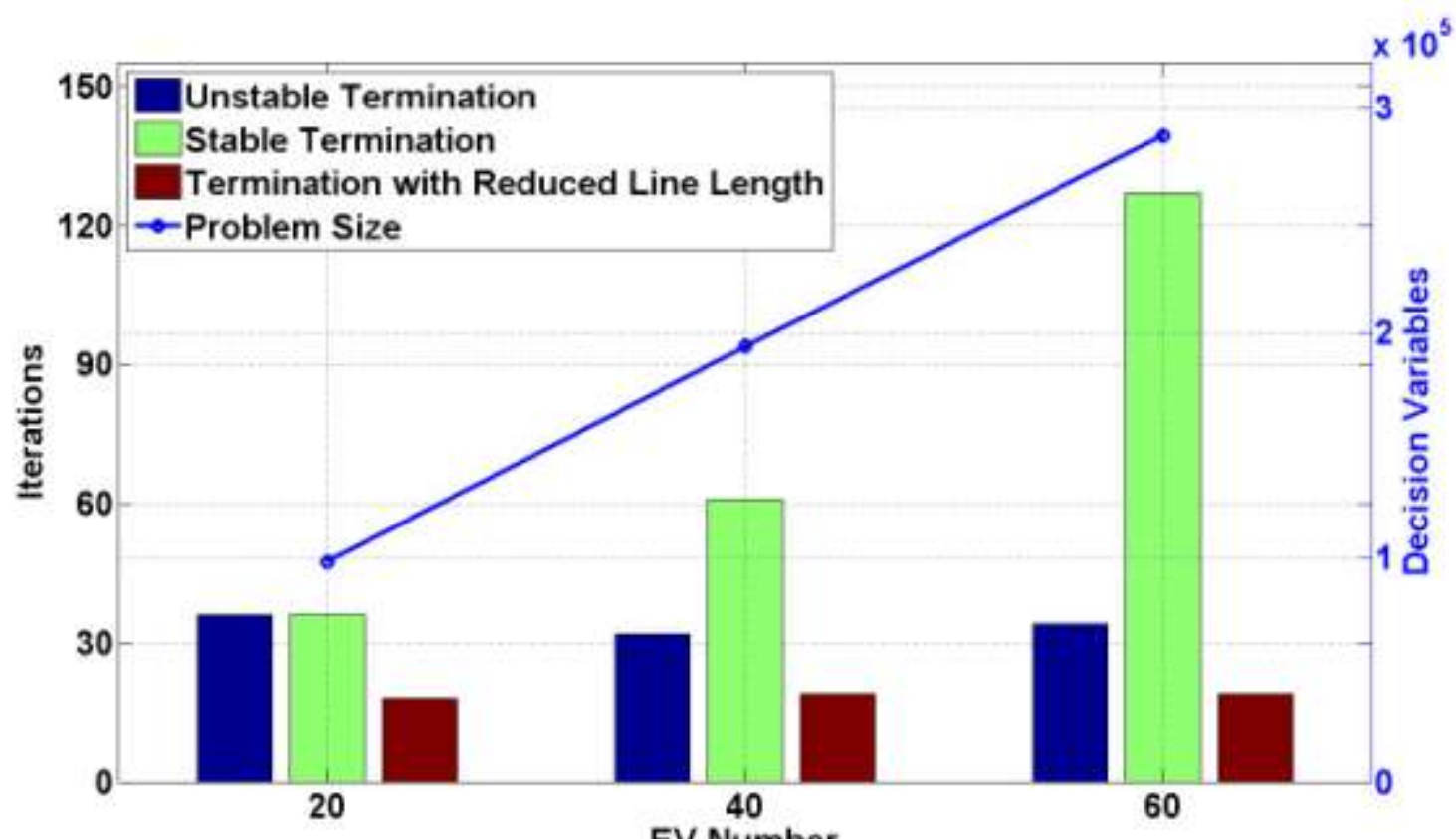

Fig.10. DSM scalability and convergence vs. the size of the problem

493 2) Stochastic vs. Deterministic. We use stochastic optimization in the proposed DSM to address the stochastic behavior of the EVs. We show the advantage of using stochastic DSM over deterministic DSM in Fig.11. To evaluate the performance of the stochastic DSM and its deterministic counterpart, we have created two test cases using each DSM. The stochastic DSM is created as using the proposed method in this paper. The deterministic DSM is constructed with the best estimation of EV user behavior, i.e. using the average value of arrival time, departure time and energy demand for each EV user. Then 200 scenarios are generated out of the 60 studied EVs. Averaged EV load mismatch are calculated, which is defined as average mismatch between the estimated EV load at each bus and the generated scenarios,

501 to study the performance of the two DSMs. As the stochastic DSM in this paper is an unbiased 502 estimation of the averaged EV load at each bus, the mismatch is zero. The averaged mismatch at each 503 bus for deterministic DSM is shown in Fig.11. The mismatches are in the day time when EVs are available 504 to SDSO. One phenomenon mismatch by using deterministic method is at 9am on bus 9. The averaged 505 active power mismatch is $24.4 \mathrm{~kW}$ while at the same time the bus 9 base active load is $77.3 \mathrm{~kW}$, which is 506 roughly $31.6 \%$ of the overall uncontrollable load. Together with other mismatches in other time under 507 various buses, traditional deterministic method creates problems for DSO to operate the distribution 508 grid. 
Two remarks on the numerical results: a) the deterministic DSM using the averaged value for each EV uncertainties still creates considerable power mismatch. There is no guarantee, for the stochastic DSM to outperform its deterministic counterpart in every scenario. However, in a long run (corresponding to larger scenarios), the stochastic DSM will outperform. b) The mismatch may continue to grow when EV number continues to scale up. Therefore, the stochastic DSM is critical for the DSO to estimate the average load under each bus with uncertainty.

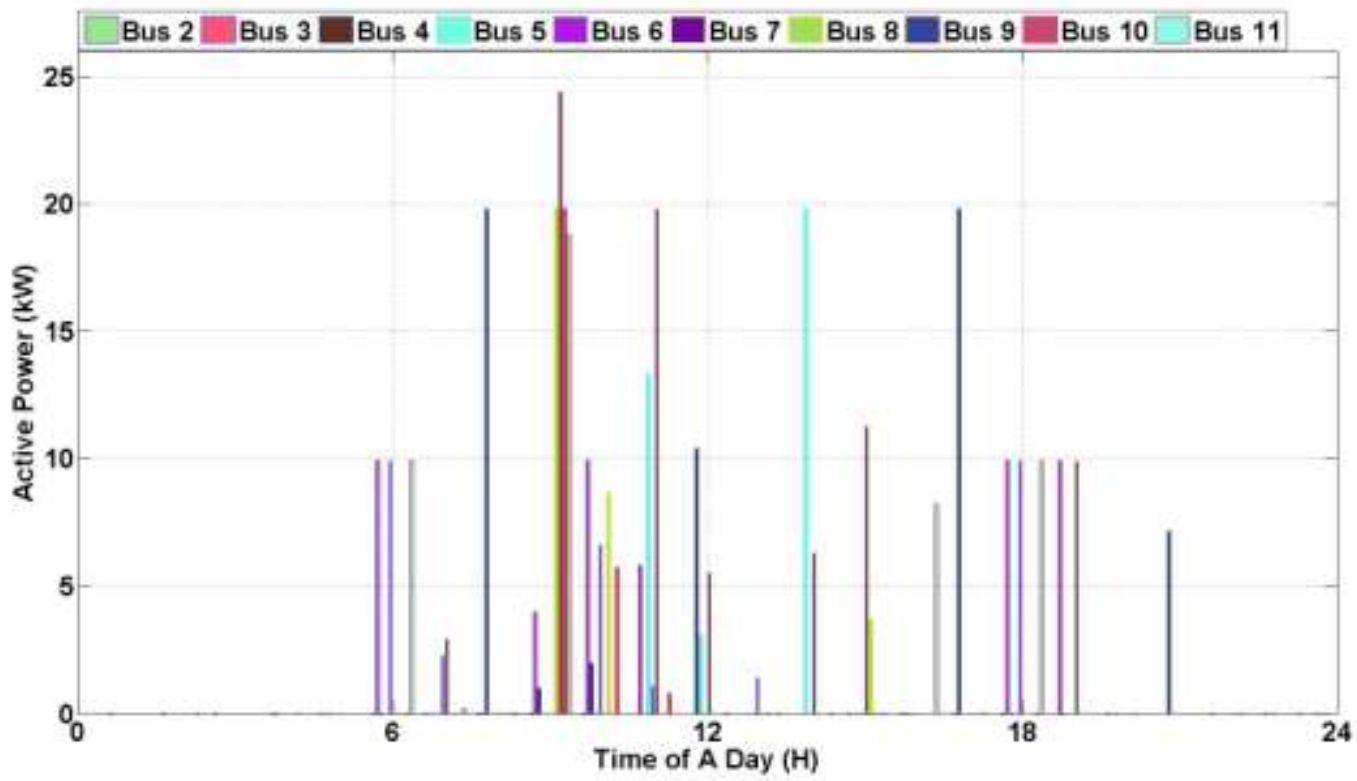

515

Fig.11. Average EV load mismatch for each bus using deterministic method

3) Lumped Model vs. Networked Model. We further study the traditional DSM which only accounts for the local effects (LDSM), i.e. minimizing the operational cost under each bus. We compare the traditional LDSM with the DSM developed in this paper in Fig.12. The two cases are created under same simulation setup with only difference in LDSM not accounting for the network and power flow constraint. As shown in Fig.12, we plot the aggregated power losses for the whole network at each time step for both DSMs. Moreover, the maximum and minimum voltage in the whole network at each time step for the two DSMs are also potted for comparisons. It is observed that the LDSM creates a much larger power loss over the distribution line. The total distribution loss is $353.9 \mathrm{kWh}$ for the studied distribution grid by using the proposed DSM for operation. On the other hand, if the traditional lumped model based DSM is applied, the total distribution losses for the load profile will be $719.8 \mathrm{kWh}$, a loss twice the size of the proposed method. Furthermore, it is also observed that the minimum voltage in the system drops to 0.92 p.u by using lumped model based DSM, while the minimum voltage in the 
proposed networked based model is regulated at 0.95 p.u.. The numerical results demonstrate the importance of switching from traditional LDSM to the proposed DSM which accounts for the network topology.

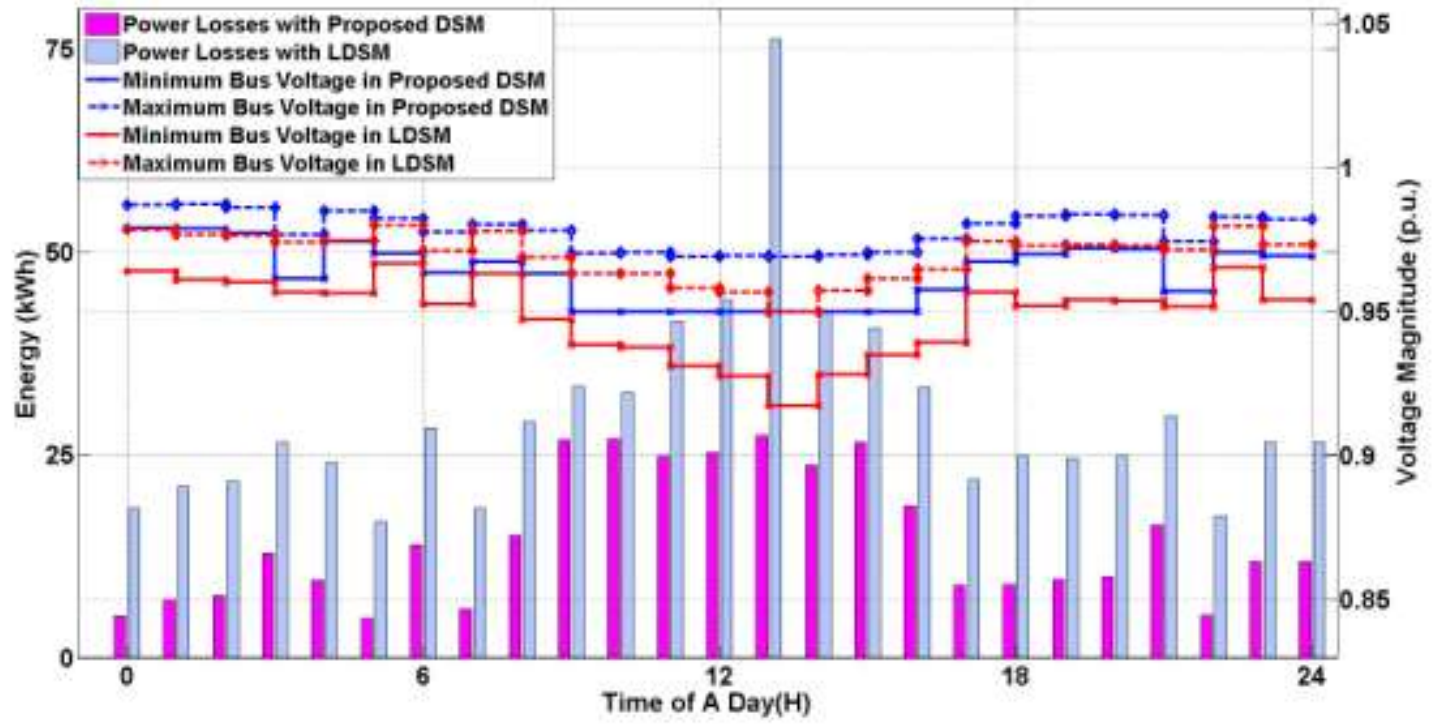

Fig.12. Comparisons of power losses and voltage regulation for DSM and LDSM

\section{Conclusions}

In this paper, we have proposed a DSM that targets at minimizing the operational cost and distribution losses over distribution networks. It accounts for the stochastic resources in the system, the scalability of increasing controllable devices, and power network physical constraints. For modeling uncertainties of stochastic resources, EVs in this paper, stochastic optimization is applied. The distribution of the arrival time, departure time and energy demands in EVs are captured using KDE. The stochastic optimization problem is numerically approximated with the help of SAA. The proposed method can be also used for modeling power system integrated with distributed resources such as solar and wind following the same modeling framework. On the other hand, distributed DSM proposed in this paper resolves the scalability issue. As SAA quickly scales up the problem size with the increase integrations of EVs, o-ADMM based DSM dispatches the computational burden originally at DSO to distributed SDSO.

545 Moreover, as a 'side effect', instead of directly communicating the power consumption to DSO, the proposed distributed DSM protects privacy of the end-users by only communicating their dual variables.

547 In the end, we also shows the necessity of switching from a traditional lumped based LDSM to the DSM 548 that accounts for network topology: the proposed DSM helps to save power losses over the distribution 
549 line and regulate the bus voltages within an acceptable range. Apart from cost and power losses

550 minimization studied in this paper, it must be pointed out that there exists other services that BESS and

551 EV could potentially provide and create greater values. It remains an open question and is to be 552 addressed in future studies. In conclusion, the methods proposed and observations made shed light on 553 future large scale load dispatch and EV/renewable integrations to distribution grids.

\section{Acknowledgement}

555 This work has been sponsored in part by a grant from the LADWP/DOE fund 20699 \& 20686, (Smart Grid 556 Regional Demonstration Project).

\section{Appendix}

558 Introduction to ADMM

559 We used Alternating Direction Method of Multipliers (ADMM) in [28] to develop our distributed DSM.

560 The ADMM solves problems in the following form:

$$
\begin{aligned}
& \text { minimize } f(x)+g(z) \\
& \text { subject to } A x+B z=c
\end{aligned}
$$

561 where $x \in \mathfrak{R}^{n}$ and $z \in \mathfrak{R}^{m}$ are decision variables. $A \in \mathfrak{R}^{p \times n}, B \in \mathfrak{R}^{p \times m}$ and $c \in \mathfrak{R}^{p}$. Assuming $f(\cdot)$ and $562 g(\cdot)$ are convex and constraints form convex set. The augmented Lagrangian of this problem is 563 presented as:

$$
L_{\rho}(x, z, y)=f(x)+g(z)+y(A x+B z-c)+\frac{\rho}{2}\|A x+B z-c\|^{2}
$$

564 Choosing a random initial value for $x, z$ and $y, A D M M$ is then formulated as the following iterations:

$$
\begin{gathered}
(x)_{k+1}=\operatorname{argmin}_{x} L_{\rho}\left(x,(z)_{k},(y)_{k}\right) \\
(z)_{k+1}=\operatorname{argmin}_{y} L_{\rho}\left((x)_{k+1}, z,(y)_{k}\right) \\
(y)_{k+1}=(y)_{k}+\rho\left(A(x)_{k+1}+B(z)_{k+1}-c\right)
\end{gathered}
$$

565 where $\rho$ is a positive number stands for step size. Through the sequential update of $x$ and $z$ the 566 searching direction of the optimum is alternating. Substituting $y=\rho u$, we derive the scaler form for the 567 ADMM as following iterations:

$$
\begin{aligned}
(x)_{k+1}= & \operatorname{argmin}_{x}\left(f(x)+\frac{\rho}{2}\left\|A x+B(z)_{k+1}-c+(u)_{k}\right\|^{2}\right) \\
(z)_{k+1}= & \operatorname{argmin}_{z}\left(g(z)+\frac{\rho}{2}\left\|A(x)_{k+1}+B z-c+(u)_{k}\right\|^{2}\right) \\
& (u)_{k+1}=(u)_{k}+A(x)_{k+1}+B(z)_{k+1}-c
\end{aligned}
$$


568 The iteration stops until it converges. If the $f(\cdot)$ and $g(\cdot)$ are convex and constraints form convex set,

569 the distributed solution is guaranteed to converge to the centralized counterpart.

570

571

572

573

574

575

576

577

578

579

580

581

582

583

584

585

586

587

588

589

590

591

592

593

594

595

596

597

598

599

600

\section{Reference}

[1] Berger L. T. and Iniewski K., Smart Grid: Applications, Communications, Security. Hoboken, NJ, USA: Wiley, Apr. 2012.

[2] Barelli, L., Bidini, G. and Bonucci, F., 2016. A micro-grid operation analysis for cost-effective battery energy storage and RES plants integration. Energy, 113, pp.831-844.

[3] Li, Q., Bai, F., Yang, B., Wang, Z., El Hefni, B., Liu, S., Kubo, S., Kiriki, H. and Han, M., 2016. Dynamic simulation and experimental validation of an open air receiver and a thermal energy storage system for solar thermal power plant. Applied Energy, 178, pp.281-293.

[4] Mukoyama, S., Matsuoka, T., Hatakeyama, H., Kasahara, H., Furukawa, M., Nagashima, K., Ogata, M., Yamashita, T., Hasegawa, H., Yoshizawa, K. and Arai, Y., 2015. Test of REBCO HTS magnet of magnetic bearing for flywheel storage system in solar power system. IEEE Transactions on Applied Superconductivity, 25(3), pp.1-4.

[5] Mocci, S., Natale, N., Pilo, F. and Ruggeri, S., 2015. Demand side integration in LV smart grids with multi-agent control system. Electric Power Systems Research, 125, pp.23-33.

[6] Zhu, Y. and Tomsovic, K., 2007. Optimal distribution power flow for systems with distributed energy resources. International Journal of Electrical Power \& Energy Systems, 29(3), pp.260-267.

[7] Nazaripouya, H., Wang, Y., Chu, P., Pota, H.R. and Gadh, R., 2015, July. Optimal sizing and placement of battery energy storage in distribution system based on solar size for voltage regulation. In 2015 IEEE Power \& Energy Society General Meeting (pp. 1-5). IEEE.

[8] International Energy Agency, Global EV Outlook, International Energy Agency, 2013, Available: http://www.iea.org/publications/globalevoutlook 2013.pdf [Online]

[9] Muñoz, E.R., Razeghi, G., Zhang, L. and Jabbari, F., 2016. Electric vehicle charging algorithms for coordination of the grid and distribution transformer levels. Energy, 113, pp.930-942.

[10] Wang, B., Huang, R., Wang, Y., Nazaripouya, H., Qiu, C., Chu, C.C. and Gadh, R., 2016, May. Predictive scheduling for Electric Vehicles considering uncertainty of load and user behaviors. In 2016 IEEE/PES Transmission and Distribution Conference and Exposition (T\&D) (pp. 1-5). IEEE

[11] Xydas, E., Marmaras, C. and Cipcigan, L.M., 2016. A multi-agent based scheduling algorithm for adaptive electric vehicles charging. Applied Energy, 177, pp.354-365.

[12] Shi, Wenbo, and Vincent WS Wong. "Real-time vehicle-to-grid control algorithm under price uncertainty." In Smart Grid Communications (SmartGridComm), 2011 IEEE International Conference on, pp. 261-266. IEEE, 2011.

[13] Sortomme, E. and El-Sharkawi, M.A., 2012. Optimal scheduling of vehicle-to-grid energy and ancillary services. IEEE Transactions on Smart Grid, 3(1), pp.351-359. 
601 [14] Lin, J., Leung, K.C. and Li, V.O., 2014. Optimal scheduling with vehicle-to-grid regulation service. IEEE Internet 602 of Things Journal, 1(6), pp.556-569.

603 [15] Saber, A.Y. and Venayagamoorthy, G.K., 2009, July. Optimization of vehicle-to-grid scheduling in constrained 604 parking lots. In 2009 IEEE Power \& Energy Society General Meeting (pp. 1-8). IEEE.

605 [16] Guille, C. and Gross, G., 2009. A conceptual framework for the vehicle-to-grid (V2G) implementation. Energy 606 policy, 37(11), pp.4379-4390.

607 [17] Bai, X. and Qiao, W., 2015. Robust optimization for bidirectional dispatch coordination of large-scale V2G. IEEE 608 Transactions on Smart Grid, 6(4), pp.1944-1954.

609 [18] Wang, Y., Wang, B., Chu, C.C., Pota, H. and Gadh, R., 2016. Energy management for a commercial building 610 microgrid with stationary and mobile battery storage. Energy and Buildings, 116, pp.141-150.

611 [19] Jian, L., Zheng, Y., Xiao, X. and Chan, C.C., 2015. Optimal scheduling for vehicle-to-grid operation with 612 stochastic connection of plug-in electric vehicles to smart grid. Applied Energy, 146, pp.150-161.

613 [20] Yang, J., He, L. and Fu, S., 2014. An improved PSO-based charging strategy of electric vehicles in electrical 614 distribution grid. Applied Energy, 128, pp.82-92.

615 [21] Crossland, A.F., Jones, D. and Wade, N.S., 2014. Planning the location and rating of distributed energy storage 616 in LV networks using a genetic algorithm with simulated annealing. International Journal of Electrical Power \& Energy 617 Systems, 59, pp.103-110.

618 [22] Sousa, T., Morais, H., Soares, J. and Vale, Z., 2012. Day-ahead resource scheduling in smart grids considering 619 vehicle-to-grid and network constraints. Applied Energy, 96, pp.183-193.

620 [23] Siano, P., Cecati, C., Yu, H. and Kolbusz, J., 2012. Real time operation of smart grids via FCN networks and 621 optimal power flow. IEEE Transactions on Industrial Informatics, 8(4), pp.944-952.

622 [24] Yadaiah, N., Kumar, A.G.D. and Bhattacharya, J.L., 2004. Fuzzy based coordinated controller for power system 623 stability and voltage regulation. Electric Power Systems Research, 69(2), pp.169-177.

624 [25] Wang, Z., Yang, K. and Wang, X., 2013. Privacy-preserving energy scheduling in microgrid systems. IEEE 625 Transactions on Smart Grid, 4(4), pp.1810-1820.

626 [26] Joo, J.Y. and Ilić, M.D., 2013. Multi-layered optimization of demand resources using lagrange dual 627 decomposition. IEEE Transactions on Smart Grid, 4(4), pp.2081-2088.

628 [27] Zhang, Y. and Giannakis, G.B., 2016. Distributed stochastic market clearing with high-penetration wind 629 power. IEEE Transactions on Power Systems,31(2), pp.895906.

630 [28] Boyd, S., Parikh, N., Chu, E., Peleato, B., and Eckstein, J. 2010, Distributed optimization and statistical learning 631 via the alternating direction method of multipliers. Foundation and Trends in Machine Learning, 3(1), pp. 1-122

632 [29] Shi, W., Xie, X., Chu, C.C. and Gadh, R., 2015. Distributed optimal energy management in microgrids. IEEE 633 Transactions on Smart Grid, 6(3), pp.1137-1146.

634 [30] Wang, L., Sharkh, S. and Chipperfield, A., 2016. Optimal coordination of vehicle-to-grid batteries and renewable 635 generators in a distribution system. Energy, pp.1-37. 
636 [31] Shi, Wenbo, Na Li, Xiaorong Xie, Chi-Cheng Chu, and Rajit Gadh. "Optimal residential demand response in 637 distribution networks." IEEE journal on selected areas in communications 32, no. 7 (2014): 1441-1450.

638 [32] Low, S.H., 2014. Convex Relaxation of Optimal Power Flow-Part I: Formulations and Equivalence. IEEE 639 Transactions on Control of Network Systems, 1(1), pp.15-27.

640 [33] Low, S.H., 2014. Convex Relaxation of Optimal Power Flow-Part II: Exactness. IEEE Transactions on Control of 641 Network Systems, 1(2), pp.177-189.

642 [34] Zhang, B. and Tse, D., 2013. Geometry of injection regions of power networks. IEEE Transactions on Power 643 Systems, 28(2), pp.788-797.

644 [35] Shapiro, A. and Dentcheva, D., 2014. Lectures on stochastic programming: modeling and theory (Vol. 16). SIAM.

645 [36] Wang, Y., Sheikh, O., Hu, B., Chu, C.C. and Gadh, R., 2014, November. Integration of V2H/V2G hybrid system 646 for demand response in distribution network. In Smart Grid Communications (SmartGridComm), 2014 IEEE 647 International Conference on (pp. 812-817). IEEE.

648 [37] Millner, A., 2010, September. Modeling lithium ion battery degradation in electric vehicles. In Innovative 649 Technologies for an Efficient and Reliable Electricity Supply (CITRES), 2010 IEEE Conference on (pp. 349-356). 650 IEEE.

651 [38] Silverman, Bernard W, Density estimation for statistics and data analysis, CRC press (1986)

652 [39] Eckstein, J., 1994. Parallel alternating direction multiplier decomposition of convex programs. Journal of 653 Optimization Theory and Applications, 80(1), pp.39-62.

654 [40] W. Kersting, "Radial distribution test feeders," in Proc. IEEE PES Winter Meet., Columbus, OH, USA, Jan. 2001, 655 pp. 908-912.

656 [41] ERCOT, Day-ahead Energy Price, [Online]. Available: http://www.ercot.com/

657 [42] Cornell University Facilities Service, Real Time Building Utility Use Data, [Online]. Available:

658 http://portal.emcs.cornell.edu/

659 [43] SAE J1772, [Online]. Available: http://standards.sae.org/wip/j1772/

660 [44] CHAdeMO society, Basic function of quick charger for the electric vehicle, Tech. Rep., 2012.

661 [45] Gurobi Optimization Inc, 2015, [Online] Available: http://www.gurobi.com/ 